\title{
'Study of the Surgical Efficacy of Laparoscopic Colectomy Vs Open Colectomy for Colon Cancer at A Tertiary Care Hospital in Mangalore'
} Dr. Abilash Carolina Abraham ${ }^{1 *}$, Dr. Erel A I Diaz ${ }^{2}$, Dr Leo Francis Tauro ${ }^{3}$

${ }^{1}$ Junior Resident in Department of General Surgery, Father Muller Medical College, Mangalore, India

${ }^{2,3}$ Professor and Unit Head, Department of General Surgery, Father Muller Medical College, Mangalore, India

DOI: $\underline{10.36347 / \text { sasjs.2020.v06i03.010 }}$

| Received: 03.11.2019| Accepted: 13.11.2019| Published: 19.03.2020

*Corresponding author: Dr. Abilash Carolina Abraham

Abstract

Original Research Article

\begin{abstract}
Aim: To study patients who have undergone laparoscopic and opencolectomy, for completeness of resection and course of stay in the hospital. Background: Laparoscopic colectomy is a relatively new advancement in treatment of colorectal malignancy and studies have shown that laparoscopic colectomy have a good outcome when compared to open colectomy in colorectal malignancy. Clinical trials have proven that LC has better short term surgical and comparable long-term oncological outcomes. Hence, comparing the open colectomy techniques with the laparoscopic methods, in terms of disease clearance, post-operative complications and overall outcome is warranted. Methods: Patient records were taken from august 2017 to august 2019, which underwent open and laparoscopic colectomy, at Father Muller Medical College Hospital were analysed. Results: 20 patients underwent laparoscopiccolectomy, and 20 underwent open colectomy. There was no significant difference in demographic data. Both the groups had a similar oncological clearance of tumor. LC group had a significant shorter hospital stay than OC group with $p$ value $<0.05$. In each group, there was no post-operative mortality, 30 day readmission or major post-operative complications. Conclusion: The outcome for patients undergoing either laparoscopic or opencolectomy was similar for demographic data, intraoperative factors, oncological clearance and histopathological factors except for a shorter hospital stay in laparoscopic colectomy.

Keywords: Surgical Efficacy Laparoscopic Colectomy.

Copyright @ 2020: This is an open-access article distributed under the terms of the Creative Commons Attribution license which permits unrestricted use, distribution, and reproduction in any medium for non-commercial use (NonCommercial, or CC-BY-NC) provided the original author and source are credited.
\end{abstract}

\section{INTRODUCTION}

Laparoscopic colectomy is a relatively new advancement in treatment of colorectal malignancy and studies have shown that laparoscopic colectomy have a good outcome when compared to open colectomy in colorectal malignancy [1]. Clinical trials have proven that LC has better short term surgical and comparable long-term oncological outcomes [2]. In addition, metaanalysis studies confirmed these findings [3]. However, there is limited data on the comparative effectiveness of LC vs open colectomy (OC).Therefore, the purpose of this study is to investigate the short-term comparative effectiveness of LC vs OC of stage I-III colon cancer patients who underwent curative surgical resection[4]. In this study, we retrospectively try to review the results of laparoscopic-assisted resection of colo-rectal carcinoma and compared them with a group of patients with resection by conventional open procedure carried out during the same period.

\section{MATERIALS AND METHODS Patients}

From august 2017 to august 2019, forty patients diagnosed with stage I-III colon cancer in our hospital. Patients were limited to those who had adenocarcinoma and underwent either curative LC or $\mathrm{OC}$ as their first course of treatment.

Study type: retrospective descriptive study

\section{INCLUSION CRITERIA}

Patients above the age of 20 years diagnosed to have stage I-III colon cancer. Patients were limited to those who had adenocarcinoma and underwent either curative LC or OC as their first course of treatment.

\section{EXCLUSION CRITERIA}

- Patients who underwent emergency surgery or those who did not have a colonic resection

- Patients were excluded if the patient had already undergone local wide excision or contiguous organ resection. 
- We also excluded patients who were either younger than age 18 years or older than 84 years at the time of diagnosis.

- Patients with tumors infiltrating the adjacent organs as detected by ultrasonography and/or computerized tomography

- Patients who did not consent to the procedure,

- Patients with intestinal obstruction or perforation.

All patients were preoperatively identified to have malignant tumor through colonoscopy and pathological biopsy. All patients gave their informed consent before the procedure. All the procedures were performed by the same operation team. The demographic data of the patients were collected. Patients' demographic variables included age and sex, American Society of Anaesthesiologists (ASA) grade; body mass index (BMI), site of tumour and type of surgery.

Important clinical variables were derived from a computerized database retrospectively which included tumor grade, lymphovascular invasion, extent of resection, adequate nodal retrieval ( $\geq 12$ lymph nodes resected during colectomy), and margin status. Operative data included type of operation and conversion to open procedure. Postoperatively complications, length of hospital stay, 30 day readmission and 30 day mortality recorded. All open and laparoscopic operations were performed by same team.

Colon cancer sites were categorized into three groups: right colon (cecum, ascending colon, and hepatic flexure of colon), transverse colon (transverse colon and splenic flexure of colon), left colon (descending colon and sigmoid colon) and rectum.

Statistical comparisons of clinical outcomes were made between the laparoscopic and open groups. Statistical analysis was done with Continuous data expressed as a median (range). To compare treatment groups, the Mann-Whitney $U$ test was applied to continuous data and the chi-square test to categorical data. A p-value of $<0.05$ was considered statistically significant.

\section{RESULTS}

\section{Comparison of demographic data}

The demographic data of the two groups are shown in Table 1. There was no significant difference in gender, age, BMI, ASA grading and tumor site between LC and OC groups.

Table-1: Comparison of demographic data

\begin{tabular}{|c|c|c|c|}
\hline & LC & OC & P value \\
\hline age ( years,median(range) $)$ & $57(37-72)$ & $54.5(42-81)$ & 0.75 \\
\hline & & & \\
\hline Gender & & & 0.525 \\
\hline male & 10 & 12 & \\
\hline female & 10 & 8 & \\
\hline & & & \\
\hline BMI( Kg/m2)( median( range)) & $26(16-35)$ & $26(17-41)$ & 1 \\
\hline & & & \\
\hline & LC & OC & 0.026 \\
\hline ASA GRADE1 & 7 & 1 & \\
\hline ASA GRADE 2 & 10 & 10 & \\
\hline ASA GRADE 3 & 3 & 9 & \\
\hline & & & \\
\hline SITE & LC & OC & 0.303 \\
\hline right colon & 6 & 5 & \\
\hline left colon & 2 & 7 & \\
\hline transverse colon & 1 & 1 & \\
\hline rectum & 11 & 7 & \\
\hline
\end{tabular}

\section{Comparison of Intraoperative factors}

There was no significant difference in type of surgery between the two groups. None of the the LC cases were converted to open.

\section{Comparison of postoperative factors}


Table-2: Showing comparison of post-operative factors

\begin{tabular}{|l|l|l|l|}
\hline & LC & OC & P value \\
\hline duration of hospital stay & $8.7+/-4.4$ & $13.5+/-5.5$ & 0.006 \\
\hline Extent of resection & $26.81 \pm 3.61$ & $24.92 \pm 5.60$ & 0.7 \\
\hline Lymph node yield & $18.85+/-11.37$ & $15.5+/-7.36$ & 0.65 \\
\hline Positive margin<5cm & 1 & 3 & 0.28 \\
\hline Lympho vascular invasion & 5 & 10 & 0.098 \\
\hline 30 day readmission & 0 & 0 & 1 \\
\hline 30 day mortality & 0 & 0 & 1 \\
\hline Postoperative complications & 0 & 0 & 1 \\
\hline
\end{tabular}

\section{Comparison of duration of hospital stay}

Time of hospital stay and time to resume early activity in LC group were which were significantly shorter than those in OC group $(P<0.05)$. The average length of hospital stay was $8.7+/-4.4$ days in LC group when compared to $18.85+/-11.37$ days in OC group. There was no significant difference in duration of hospital stay between primary anastomosis or stoma in both groups.

\section{Comparison of oncological clearance}

The lengths of the specimens in LC and OC groups were $26.81 \pm 3.61 \mathrm{~cm}$ and $24.92 \pm 5.60 \mathrm{~cm}$ respectively and did not show any statistically significant difference between both groups .The average number of total lymph node yield, including epicolic and paracolic lymph nodes, intermediate lymph nodes and principal lymph nodes in LC group was $18.85+/-$ 11.37 and OC group was 15.5+/-7.36 and had no statistically significant difference. The number of cases with positive surgical margin was not significantly different between LC group and OC group.

\section{Comparison of histopathological factors}

The number of cases with lymphovascular invasion in LC group compared to OC group was not statistically significant. All tumors were adenocarcinoma with maximaum number of cases of well differentiated adenocarcinoma. There was no significant difference in grade of tumor between both the groups.

\section{Comparison of post-operative recovery}

All the patients were followed-up for 30 days. No patient had 30 day mortality, 30 day readmission in either LC or OC group. No major postoperative complications were found in either group.

\section{DISCUSSION}

Laparoscopic colorectal surgery, especially for rectosigmoid cancer, is becoming increasingly popular with decent initial results [5]. But due to relatively complicated anatomy and much higher requirements for surgery technique, laparoscopic colectomy is developing relatively slow compared to open colectomy [6]. Laparoscopic colectomy for colic malignancy has not been generally accepted, and it is still controversial about the operative safety, oncological results and longterm survival rate [7].This homochronous clinical contrast study compared the clinical effects of laparoscopic and traditional open colectomy, so as to investigate the applicability of laparoscopic surgery for colon cancer.

Our study did not show any statistically significant difference between LC and OC group with regards to demographic data -gender, age, BMI, ASA grading and tumor site.

With regards to intraoperative factors there was no significant difference in type of surgery between the two groups. None of the the LC cases were converted to open.

Time of hospital stay and time to resume early activity in LC group were which were significantly shorter than those in OC group $(P<0.05)$.

In terms of oncological clearance the lengths of the specimens in LC and OC groups did not show any statistically significant difference between both groups. The average number of total lymph node yield, including epicolic and paracolic lymph nodes, intermediate lymph nodes and principal lymph nodes had no statistically significant difference. The number of cases with positive surgical margin was not significantly different between LC group and OC group. The number of cases with lymphovascular invasion in LC group compared to OC group was not statistically significant. All tumors were adenocarcinoma and there was no significant difference in grade of tumor between both the groups.

In our study none of the patients had 30 day mortality, 30 day readmission in either LC or OC group. No major postoperative complications were found in either group. Study done by steven kwon et al. [8] after adjustment (for age, sex, albumin levels, diabetes, body mass index, comorbidity index, cancer diagnosis, year, hospital bed size, and urban vs rural location), the risk of transfusions, wound infections and composite of adverse events were all significantly lower with laparoscopy colectomy.

A study done by Antonio Lacy et al. [9] 219 patients took part in the study (111 LAC group, 108 OC group). Patients in the LAC group recovered faster than 
those in the OC group, with shorter peristalsis-detection $(\mathrm{p}=0.001)$ and oral-intake times $(\mathrm{p}=0 \cdot 001)$, and shorter hospital stays $(p=0 \cdot 005)$. Morbidity was lower in the LAC group $(\mathrm{p}=0 \cdot 001)$, although LAC did not influence perioperative mortality.

Our study showed that in LC group patients the duration of hospital stay were shorter compared to the OC group. Compared to laparoscopic rectal cancer surgery, a similar study done by nelson et al. [10] the Perioperative recovery was faster in the laparoscopicsurgery group than in the open-colectomy group, as reflected by a shorter median hospital stay (five days vs. six days, $\mathrm{P}<0.001)$ and briefer use of parenteral narcotics (three days vs. four days, $\mathrm{P}<0.001$ ) and oral analgesics (one day vs. two days, $\mathrm{P}=0.02$ ). The rates of intraoperative complications, 30-day postoperative mortality, complications at discharge and 60 days, hospital readmission, and reoperation were very similar between groups.

\section{CONCLUSION}

From our study, we conclude that for carcinomacolon, both laparoscopic and open methods were equally efficient methods, with almost similar demographic data, oncological clearance and postoperative complications. Our study also concludes that LC group has significantly shorter hospital stay when compared to OC group. However for better results. A larger, randomized control trial has to be done to evaluate the long term outcome and benefits of laparoscopic colectomy as opposed to open colectomy.

\section{REFERENCES}

1. Reza MM, Blasco JA, Andradas E, Cantero R, Mayol J. Systematic review of laparoscopic versus open surgery for colorectal cancer. British Journal of Surgery: Incorporating European Journal of Surgery and Swiss Surgery. 2006 Aug;93(8):921-8.

2. Schwenk W, Haase O, Neudecker JJ, Müller JM. Short term benefits for laparoscopic colorectal resection. Cochrane database of systematic reviews. 2005(2).

3. Jacobs M, Verdeja JC, Goldstein HS. Minimally invasive colon resection (laparoscopic colectomy). Surgical laparoscopy \& endoscopy. 1991 Sep;1(3):144-50.

4. Guillou PJ, Darzi A, Monson JR. Experience with laparoscopic colorectal surgery for malignant disease. Surgical oncology. 1993 Jan 1;2:43-9.

5. Berends F, Kazemier G, Bonjer H, Lange J. Subcutaneous metastases after laparoscopic colectomy. The Lancet. 1994 Jul 2;344(8914):58.

6. Kazemier G, Bonjer HJ, Berends FJ, Lange JF, Rae D, Bowyer R, Wharton R, Paterson I. Port site metastases after laparoscopic colorectal surgery for cure of malignancy. British Journal of Surgery. 1995 Aug;82(8):1141-2.

7. Lohsiriwat V, Lohsiriwat D, Chinswangwatanakul V, Akaraviputh T, Lert-Akyamanee N. Comparison of short-term outcomes between laparoscopicallyassisted vs. transverse-incision open right hemicolectomy for right-sided colon cancer: a retrospective study. World journal of surgical oncology. 2007 Dec;5(1):49.

8. Care TS, Collaborative OA, Kwon S, Billingham R, Farrokhi E, Florence M, Herzig D, Horvath K, Rogers T, Steele S, Symons R. Adoption of laparoscopy for elective colorectal resection: a report from the Surgical Care and Outcomes Assessment Program. Journal of the American College of Surgeons. 2012 Jun 1;214(6):909-18.

9. Lacy AM, García-Valdecasas JC, Delgado S, Castells A, Taurá P, Piqué JM, Visa J. Laparoscopy-assisted colectomy versus open colectomy for treatment of non-metastatic colon cancer: a randomised trial. The Lancet. 2002 Jun 29;359(9325):2224-9.

10. Dean PA, Beart Jr RW, Nelson H, Elftmann TD, Schlinkert RT. Laparoscopic-assisted segmental colectomy: early Mayo Clinic experience. InMayo Clinic Proceedings 1994 Sep 1 (Vol. 69, No. 9, pp. 834-840). Elsevier. 